\title{
Expression of 58-kD Microspherule Protein (MSP58) is Highly Correlated with PET Imaging of Tumor Malignancy and Cell Proliferation in Glioma Patients
}

\author{
Wei Lin ${ }^{a, b}$ Shu-hui Dai ${ }^{a}$ Tao Chen ${ }^{a, e}$ Nobuyuki Kawai ${ }^{b}$ Keisuke Miyake \\ Masaki Okada ${ }^{b}$ Reiji Habac Yuka Yamamoto ${ }^{d}$ Takashi Tamiya ${ }^{b}$ Zhou Fei $^{\text {a }}$ \\ aDepartment of Neurosurgery, Xijing Hospital, Fourth Military Medical University, Xi'an, China; \\ bDepartment of Neurological Surgery, Faculty of Medicine, Kagawa University, Kita-gun, Japan; \\ 'Department of Diagnostic Pathology, Faculty of Medicine, Kagawa University, Kita-gun, Japan; \\ dDepartment of Radiology, Faculty of Medicine, Kagawa University, Kita-gun, Japan; eDepartment of \\ Neurosurgery, The 123th Hospital of PLA, Bengbu, China
}

\section{Key Words}

Fluorothymidine $(\mathrm{FLT}) \cdot$ Glioma $・ \mathrm{MSP} 58$ • Positron emission tomography (PET) • Proliferation

\begin{abstract}
Background/Aims: The nucleolar 58-kDa microspherule protein (MSP58) has important transcriptional regulation functions and plays a crucial role in the tumorigenesis and progression of cancers. $3^{\prime}$-deoxy-3'- $\left.{ }^{18} \mathrm{~F}\right]$ fluorothymidine (FLT) has emerged as a promising positron emission tomography (PET) tracer for evaluating tumor malignancy and cell proliferation. Methods: In the present study, the expression of MSP58 was evaluated by immunohistochemistry and the corresponding PET image was examined using FLT-PET in 55 patients with various grades of gliomas. Results: The immunoreactivity score (IRS) of MSP58 increased with tumor grade with grade IV gliomas exhibiting the highest expression and showed a highly significant positive correlation with the Ki-67 index $(r=0.65, P<0.001)$. The IRS of MSP58 in the tumor showed a highly significant positive correlation with corresponding FLT uptake value $(r=0.61, P<$ 0.001 ). The correlation between MSP58 expression and glioma malignancy was also confirmed by immunofluorescence, RT-PCR and western blot analysis. FLT uptake value also exhibited a highly significant positive correlation with the Ki-67 index $(r=0.59, P<0.001)$. KaplanMeier analysis revealed that MSP58 expression has a significant prognostic ability for the overall survival time similar to that found in the uptake value of FLT-PET. Conclusion: These results indicate that MSP58 plays an important role in cell proliferation and will be one of the potential candidates of molecular therapy targeting proliferation. FLT-PET might be used as an early measure of treatment response in the proliferation-targeted therapy.
\end{abstract}

W. Lin, S.-h. Dai and T. Chen contributed equally to this work.

Dr. Zhou Fei and Dr. Takashi Tamiya

KARGER
Department of Neurosurgery, Xijing Hospital, Fourth Military Medical University, No. 127 ChangLe West Road, Xi'an, Shaanxi Province 710032, (China); and Department of Neurological Surgery, Faculty of Medicine, Kagawa University, Kita-gun, (Japan)

E-Mail fmmuzhoufei@163.com or Tamiya_kagawa@163.com 


\section{Introduction}

Gliomas are the most common primary brain tumors arising in the central nervous system. Although various methods have been proposed to improve the diagnostic and prognosis evaluation ability in patients with gliomas, a significant rate of misjudge still exists. Identification of new key molecules and development of new methods would help to diagnose and evaluate the prognosis of the patients accurately and have significance for treating this terrible disease.

The nucleolar 58-kDa microspherule protein (MSP58) also referred to microspherule protein 1 (MCRS1) is a 462-amino acid protein that contains a nucleolar localization signal, a nuclear localization signal, a coiled-coil domain and a fork head-associated (FHA) domain that is also considered to be the phosphopeptide binding module [1,2]. Several studies have demonstrated that MSP58 interacts with various proteins that play crucial roles in transcriptional regulation functions [3-6]. Our previous study showed that MSP58 is important in biological progression of glioma cell lines and correlates with the WHO tumor grade and overall survival in patients with glioma $[7,8]$. Accumulating evidence suggests that the expression of MSP58 has key functions in tumorigenesis and malignant transformation in gliomas and might be a novel prognostic biomarker that could be used as an adjunct to the WHO tumor grade to direct the therapy regimen for an individual patient.

Positron emission tomography (PET) imaging can provide non-invasive quantitative data regarding energy use, cellular signaling, transport, and proliferation of cancers. Among several radiotracer molecules applied, Shields et al. have developed 3'-deoxy-3'-[18F] fluorothymidine (FLT) as a PET tracer to image cell proliferation in vivo [9]. They established FLT as an analogue substrate of thymidine, which is phosphorylated by the thymidine kinase- 1 (TK1) in the cells. TK1 activity has been reported to be proportional to the proliferative activity of the tumor cells and can be imaged by FLT as selective substrate for TK1 that converts FLT to its nucleotide monophosphate [10]. The application of FLT phosphorylation as a marker of cell proliferation is based on the assumption that cellular FLT trapping is a representation of thymidine incorporation into DNA $[9,11]$. In clinical studies, FLT has been validated to assess tumor malignancy and cell proliferation accurately in gliomas [12-15].

In the present study, our hypothesis is that there is a possible relationship between the expression of MSP58 and PET imaging of tumor biological aggressiveness. To verify this hypothesis, the expression of MSP58 in the tumors was evaluated by immunohistochemistry and the corresponding PET image was examined using FLT-PET in 55 patients with various grades of gliomas. We also conducted a survival analysis to evaluate whether the expression of MSP58 and the corresponding PET images can provide similar valuable information in the prognosis of these patients.

\section{Materials and Methods}

\section{Patients and tumor}

Between May 2009 and November 2013, 37 patients with newly diagnosed (18 men and 19 women aged 5-86 years; mean age, $50.0 \pm 18.5$ years) and 18 patients with recurrent ( 9 men and 9 women aged 6-71 years; mean age, $45.2 \pm 17.7$ years) malignant gliomas were preoperatively examined with FLT-PET. All the patients underwent conventional MR imaging with contrast enhancement (Gd-DTPA) before the surgery. Histopathology was performed on tissue specimens obtained by stereotactic biopsy $(n=4)$ or resection (n = 51). All tumors were graded by the 2007 World Health Organization (WHO) classification of the tumors of the central nervous system. In 37 newly diagnosed gliomas, 6 tumors were graded as grade II (4 diffuse astrocytoma, 1 oligoastrocytoma, 1 ependymoma), 12 tumors were graded as grade III gliomas (7 anaplastic astrocytoma, 4 anaplastic oligoastrocytoma, 1 anaplastic ependymoma) and 19 tumors were graded as grade IV gliomas (16 glioblastoma, 2 glioblastoma with oligodendrogliomal component, 1 gliosarcoma). In 18 recurrent gliomas, 2 tumors were graded as grade II (2 diffuse astrocytoma), 4 tumors were graded as grade III gliomas ( 3 anaplastic oligoastrocytoma, 1 anaplastic ependymoma) and 12 tumors were graded as grade IV gliomas (10 glioblastoma, 1 glioblastoma with oligodendrogliomal component, 1 gliosarcoma). 


\section{Cellular Physiology Cell Physiol Biochem 2016;38:635-645 \begin{tabular}{cc|c|c|c|} 
DOI: 10.1159/000438656 & (0) 2016 The Author(s). Published by S. Karger AG, Basel \\
and Biochemistry & Published online: February 08, 2016 www.karger.com/cpb
\end{tabular} \\ Lin et al.: MSP58 and PET Imaging in Glioma Patients}

The cellular proliferation activity of the tumor was determined by measuring the Ki-67 labeling index obtained by immunohistochemical staining with anti-Ki-67 antibody. Immunostained slides were examined at high-power magnification $(\times 400)$. The percentage of tumor cells that stained positively for Ki-67 antigen was measured in the area containing the largest number of positive tumor cells and was regarded as representative of the tumor proliferation activity. We evaluated survival time in only newly diagnosed patients $(n=37)$ and overall survival (OS) time was calculated from the date of histological diagnosis to the date of death or the last date of follow-up (January 2014).

\section{MET and FLT synthesis and PET acquisition}

The use of FLT as a PET tracer was approved by the Kagawa University Faculty of Medicine Human Subjects Ethical Committees and informed consent was obtained from all patients with written consent received before the PET examination. 18F for labeling FLT was produced using HM-18 cyclotron (Sumitomo Heavy Industries Co., Tokyo, Japan). FLT was synthesized using the method described by Machulla et al. [16] and the radiochemical purity of the produced FLT was $>95 \%$. PET examinations were performed using an ECAT EXACT HR+ scanner (Siemens/CTI, Knoxville, TN, USA) or a Biograph mCT PET/CT scanner (Siemens/ CTI, Knoxville, TN, USA) in the three-dimensional acquisition mode. No special dietary instructions were given to the patients before PET examination. Images were acquired with patients in the supine position, resting, with their eyes closed. Attenuation correction was performed using ${ }^{68} \mathrm{Ge}$ rod sources rotating around the head (ECAT EXACT HR+) or CT maps (Biograph mCT PET/CT). A dose of 106-393 MBq (mean, $285 \pm 58 \mathrm{MBq}$ ) of FLT was injected intravenously. Regional emission images of the brain were obtained for 5 min, beginning 40 min after FLT injection.

\section{PET Data Analysis}

MET and FLT uptakes in the brain lesion were semi-quantitatively assessed by evaluating the standardized uptake value (SUV). The region of interest (ROI) was set manually by an observer around the hottest area of each lesion. The maximum value of SUV (SUVmax) was regarded as the representative value of each lesion. To calculate the lesion-to-normal tissue count density $(\mathrm{L} / \mathrm{N})$ ratios, ROI was set on the normal brain parenchyma (usually contralateral normal cerebral tissue excluding ventricles) and the mean value of SUV (SUVmean) was calculated. The L/N ratio was determined by dividing the SUVmax of the lesion with the SUVmean of the normal brain tissue.

\section{Immunohistochemistry of MSP58}

Immunohistochemical assays were performed with the streptavidin-peroxidase methods as follows. Formalin-fixed, paraffin-embedded tissue blocks were cut into $4-\mu \mathrm{m}$ serial sections. After being routinely deparaffinized and dehydrated, sections were subjected to antigen retrieval by microwave treatment in $0.01 \mathrm{M}$ citrate buffer ( $\mathrm{pH} \mathrm{6.0)}$ ) for $15 \mathrm{~min}$. Endogenous peroxidase activity was blocked with $0.3 \% \mathrm{H} 2 \mathrm{O} 2$ for $30 \mathrm{~min}$. After washing three times with $0.1 \mathrm{M}$ phosphate-buffered saline (PBS) for a total of $15 \mathrm{~min}$, staining was performed using the rabbit polyclonal antibody for MSP58 (HPA039057, 1/100; Atlas Antibodies AB., Stockholm, Sweden). The primary antibody was incubated overnight at $4^{\circ} \mathrm{C}$ in a humidified chamber. After washing three times with $0.1 \mathrm{M}$ PBS for a total of $15 \mathrm{~min}$, the sections were reacted with diluted biotinylated secondary antibody for $30 \mathrm{~min}$ at room temperature and rinsed three times with $0.1 \mathrm{M}$ PBS for a total of $15 \mathrm{~min}$, followed by incubation with a Vectastain Elite ABC reagent diluted in 0.1 M PBS for $30 \mathrm{~min}$ at room temperature. After rinsing with $0.1 \mathrm{M}$ PBS, the sections were developed in a staining solution containing 0.03\% 3,3'-diaminobenzidine tetrahydrochloride (DAB peroxidase substrate kit, SK4100; Vector Laboratories, Burlingame, CA) for 5-10 min at room temperature. Sections were examined under a light microscope to check that the staining was satisfactory. To facilitate cytoplasmic or nuclear visualization of the immunostained product, Mayer's haematoxylin was used for counterstaining. As negative immunohistochemical controls, consecutive sections were incubated with normal rabbit or mouse serum. No specific immunopositive staining was detected in these control sections.

\section{Semiquantitative analysis of immunohistochemistry}

Microscopic analysis was performed by an observer (W.L.) without prior knowledge of radiological and clinical information. The immunoreactivity of MSP58 in tumor cells was graded semiquantitatively. The percentage of immunoreactive positive tumor cells, the staining intensity, and the resulting immunoreactivity 


\section{Cellular Physiology Cell Physiol Biochem 2016;38:635-645

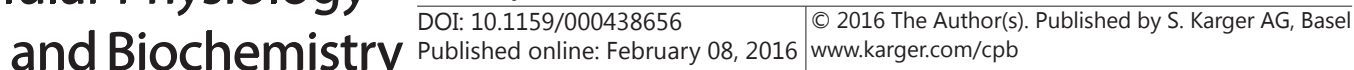 \\ Lin et al.: MSP58 and PET Imaging in Glioma Patients}

score (IRS) were assessed following a previously described technique with minor modification [17]. Briefly, the most strongly stained tumor cell area of each section of more than 1,000 tumor cells was viewed in high-powered fields at $\times 400$ magnification. The percentages of MSP58 cells were calculated by dividing the numbers of positively stained tumor cells by the total numbers of tumor cells observed. The sections that contained $1-25 \%, 26-50 \%, 51-75 \%$ and $76-100 \%$ positive cells were scored as 1, 2, 3, and 4, respectively (Fig. 1A-D). The staining intensity was graded as no staining, weak, moderate or strong, and scored as 0, 1, 2 and 3, respectively (Fig. 1E-H). Because of the heterogeneity of the staining intensity of the tumor cells, the intensity of staining of each section was determined according to the staining intensity of most of the cells. The IRS was determined by multiplying the values for staining intensity and the values for the percentage of immunoreactive tumor cells.

\section{Quantitative real-time RT-PCR}

Total RNA was isolated from human brain and brain tumour tissues using TRIZOL reagent (Invitrogen, Carlsbad, CA, USA) according to the manufacturer's protocol. Total RNA ( $2 \mu \mathrm{g})$ was reverse transcribed with reverse transcriptase (Fermentas Vilnius, Lithuania). The first strand cDNA was used as the template for real-time quantitative PCR analysis. $\beta$-actin cDNA was used as an internal control to normalize variances. The real-time quantitative PCR primers were designed using the Primer Express software (Applied Biosystems, Foster City, CA, USA). The sequences used were as followed: MSP58: F: AGG CTA TTG CAG CCA TCC AGAG, R: CTG TGC AGC AGG TCC TGGAA; $\beta$-actin: F: ATC ATG TTT GAG ACC TTC AACA, R: CAT CTC TTG CTC GAA GTCCA. The thermal cycling conditions were as following: $95^{\circ} \mathrm{C}$ for $5 \mathrm{~min}$., followed by 45 cycles of $95^{\circ} \mathrm{C}$ for $30 \mathrm{sec}$., $60^{\circ} \mathrm{C}$ for $30 \mathrm{sec}$. The $2-\Delta \Delta \mathrm{Ct}$ equation was applied to calculate the relative expression of the genes in tumor samples versus the median of normal brain tissues, where $\Delta \mathrm{Ct}=\mathrm{Ct}$ gene $-\mathrm{Ct} \beta$-actin, and $\Delta \Delta \mathrm{Ct}=\mathrm{Ct}$ tumor - mean $\Delta \mathrm{Ct}$ normal brain tissues, To ensure specificity of the PCR product amplification, the melting curves for standard and sample products were analyzed.

\section{Western blot analysis}

Equivalent amounts of protein ( $40 \mu \mathrm{g}$ per lane) were loaded and separated by $10 \%$ SDS-PAGE gels, and transferred to polyvinylidene difluoride (PVDF) membranes. Membranes were blocked with $5 \%$ nonfat milk solution in tris-buffered saline with $0.1 \%$ Triton X-100 (TBST) for $1 \mathrm{~h}$, and then incubated overnight at $4^{\circ} \mathrm{C}$ with the primary antibody dilutions in TBST. The MSP58 (sc-376569) and $\beta$-actin (sc-130300) antibody obtained from Santa Cruz Biotechnology. After that the membranes were washed and incubated with secondary antibody for $1 \mathrm{~h}$ at room temperature. Immunoreactivity was detected with Super Signal West Pico Chemiluminescent Substrate (Thermo Scientific, Rockford, IL, USA). An analysis software named Image J (Scion Corporation) was used to quantify the optical density of each band.

\section{Statistical analysis}

All parameters are presented as mean \pm standard deviation. Statistical analyses were performed in all tumors $(n=55)$ except survival estimate in that only newly diagnosed tumors $(n=37)$ were evaluated. The non-parametric IRSs of MSP58 were compared among various grades of glioma by Steel-Dwass test. The parametric $\mathrm{T} / \mathrm{N}$ ratios of FLT uptake in the tumor were compared among various grades of glioma by Bonferoni-Dunn test. The correlations between the IRSs of MSP58 in the tumor and the T/N ratios of FLT, and the Ki-67 index were analyzed using the non-parametric Spearman's rank test. Linear regression analysis was used to determine whether FLT T/N ratio was related to the Ki-67 index. Survival estimate was performed according to the Kaplan-Meier method between the patients with low (2-8) and high (9-12) IRS of MSP58 in the tumor. Overall survival time was also compared between the patients with low $(\leq 5)$ and high (> 5) T/N ratio of FLT. All the statistical computations were performed using SPSS version 15.0 (IBM Corporation, Chicago, IL, USA). Probability values of $<0.05$ were considered statistically significant.

\section{Results}

MSP58 expression and tumor grade and Ki-67 index

As shown in Fig. 1, MSP58 was mainly expressed in the nucleus of glioma cells, though a small fraction of MSP58 was expressed in the cytoplasm. Moderate to strong MSP58 

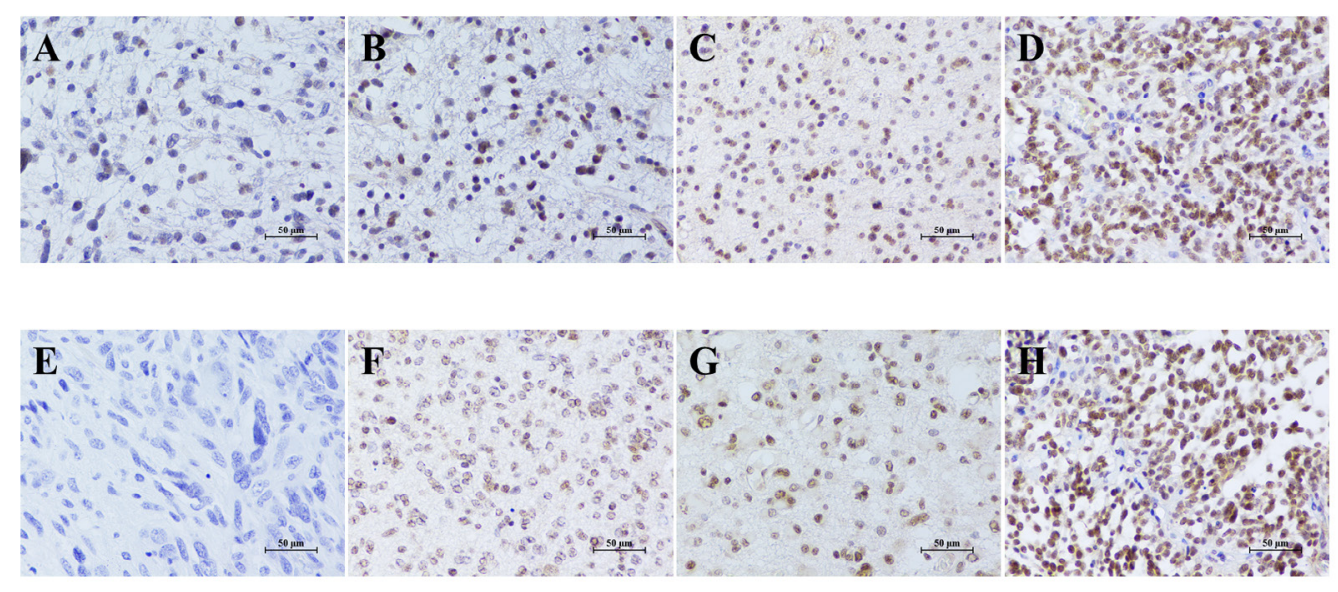

Fig. 1. Representative cases of semi-quantitative grading of staining intensity for MSP58; (A) 0: no staining, (B) 1: weak staining, (C) 2: moderate staining, (D) 3: strong staining. Representative cases of semi-quantitative grading of positive proportion for MSP58; (E) 1: 1-25\% (negative control), (F) 2: 26-50\%, (G) 3: 51-75\%, (H) 4: 76-100\%.

Fig. 2. (A) Immunoreactivity score (IRS) of MSP58 in different grades of gliomas. The average IRS of MSP58 is significantly different between grade II and grade III gliomas $(P<0.01)$, and grade III and grade IV gliomas $(P<0.001)$. (B) The IRS of MSP58 in the tumor shows a significant positive correlation with the Ki-67 index $(r=0.65$, $P<0.001$ ).
A

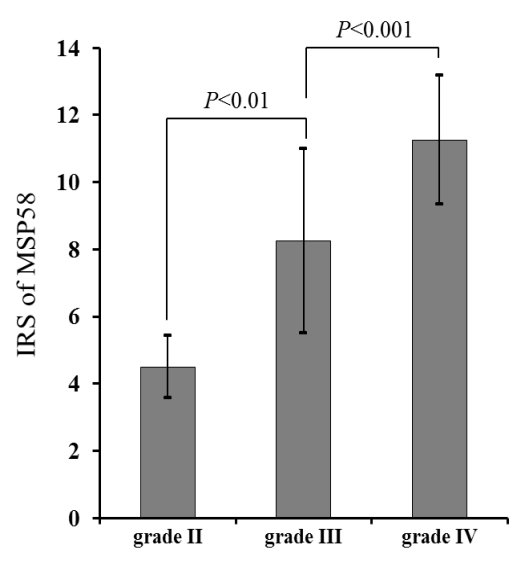

B

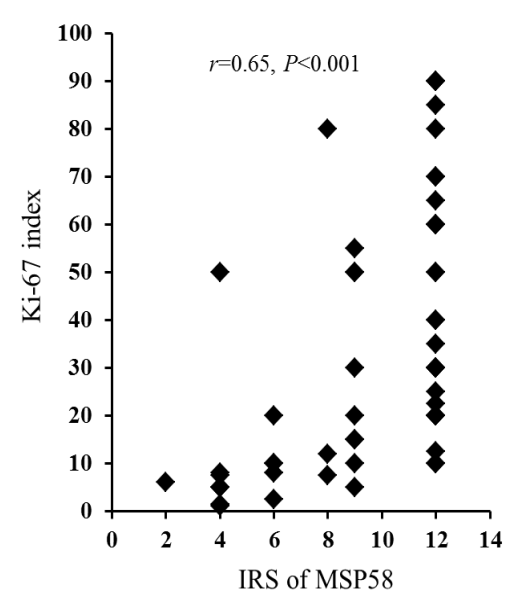

expression (staining intensity of 2 or 3 ) was seen almost all cases (54 of 55 cases). Only 1 anaplastic astrocytoma exhibited weak MSP58 immunostaining (staining intensity of 1). The average IRS of MSP58 in grade II $(n=8)$, grade III $(n=16)$, and grade IV $(n=31)$ gliomas were $4.5 \pm 0.9,8.3 \pm 2.7$, and $11.3 \pm 1.9$, respectively (Fig. 2A). The IRS of MSP58 immunoreactivity increased with tumor grade with grade IV gliomas exhibiting the highest expression, and this finding was statistically significant $(\mathrm{P}<0.001$, Kruskal-Wallis). The expression of MSP58 (IRS) was significantly different between grade II and grade III gliomas $(\mathrm{P}<0.01)$ and grade III and grade IV gliomas $(\mathrm{P}<0.001)$ (Fig. 2A). The immunohistochemical expression of MSP58 in the tumor showed a highly significant positive correlation with the Ki-67 index ( $\mathrm{r}$ $=0.65, \mathrm{P}<0.001$, non-parametric Spearman's rank test) (Fig. 2B).

Expression of MSP58 in tumor samples

To further confirm the correlation between MSP58 expression and tumor grade, we detected MSP58 expression using immunofluorescence. The results showed that MSP58 was 


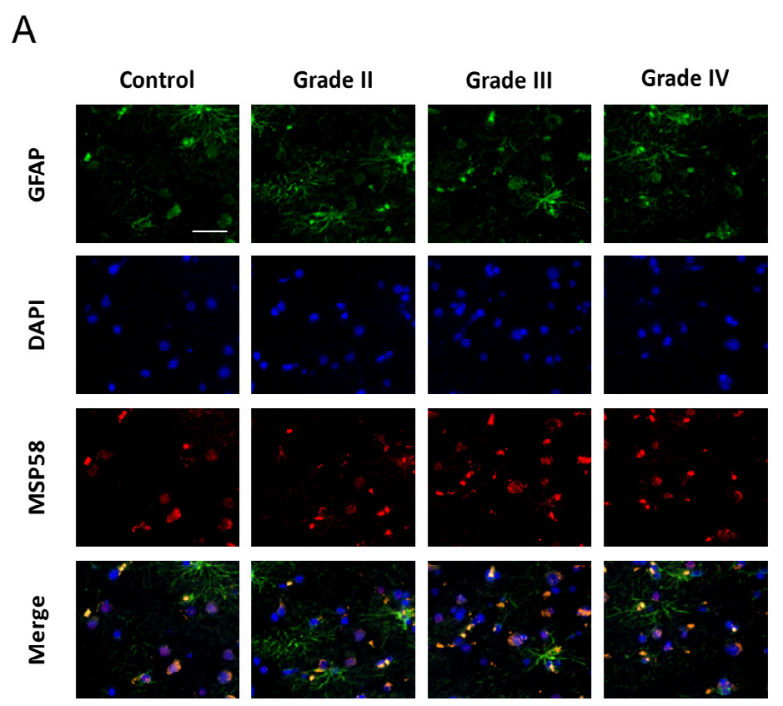

B

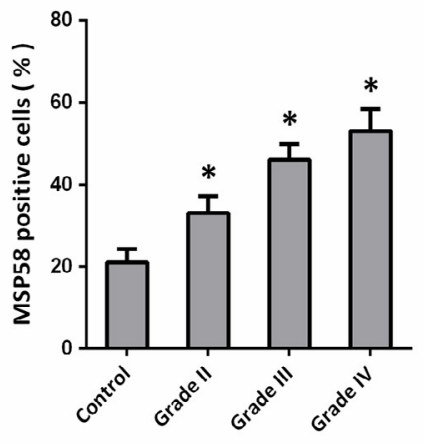

Fig. 3. (A) Representative cases of immunofluorescence of MSP58 in tumor samples. (B) The number of MSP58-positive cells in tumor samples. Scar bars: $100 \mu \mathrm{m}$.

A

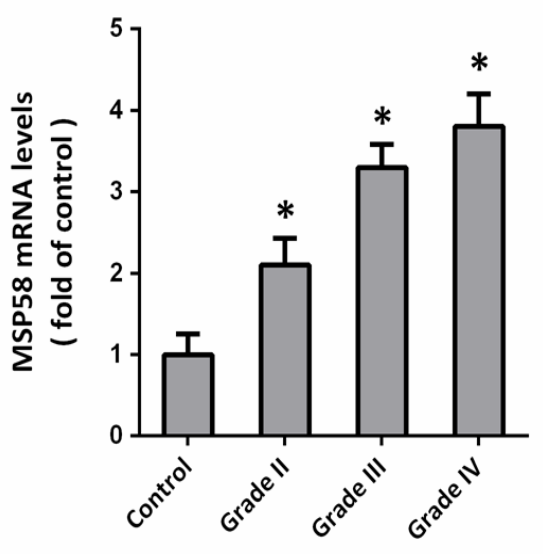

B

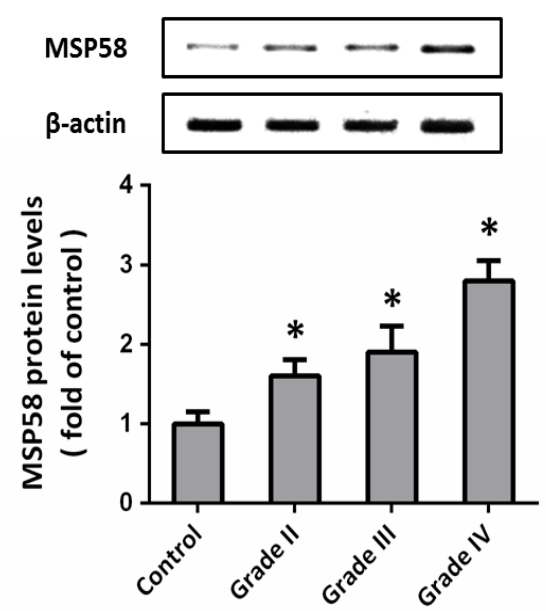

Fig. 4. (A) The expression of MSP58 mRNA in control and tumor samples. (B) The expression of MSP58 protein in control and tumor samples.

mainly expressed in the nucleus of glioma cells, which was identified by staining with GFAP (Fig. 3A). The number of MSP58 positive cells was significantly different between control and glioma cells, and it was increased in a grade dependent manner in glioma cells (Fig. 3B). In addition, we also detected the expression of MSP58 at mRNA and protein levels in tumor samples using RT-PCR and western blot analysis. The results showed that the expression of MSP58 mRNA and protein were both significantly increased in glioma cells compared with control cells, and MSP58 is increased in gliomas with high grade (Fig. 4A and 4B).

\section{Correlation between MSP58 expression and FLT uptake}

The average T/N ratio of FLT in grade II $(n=8)$, grade III $(n=16)$, and grade IV $(n=31)$ gliomas were $1.1 \pm 0.4,4.2 \pm 2.6$, and $9.2 \pm 5.4$, respectively. FLT T/N ratio was significantly 


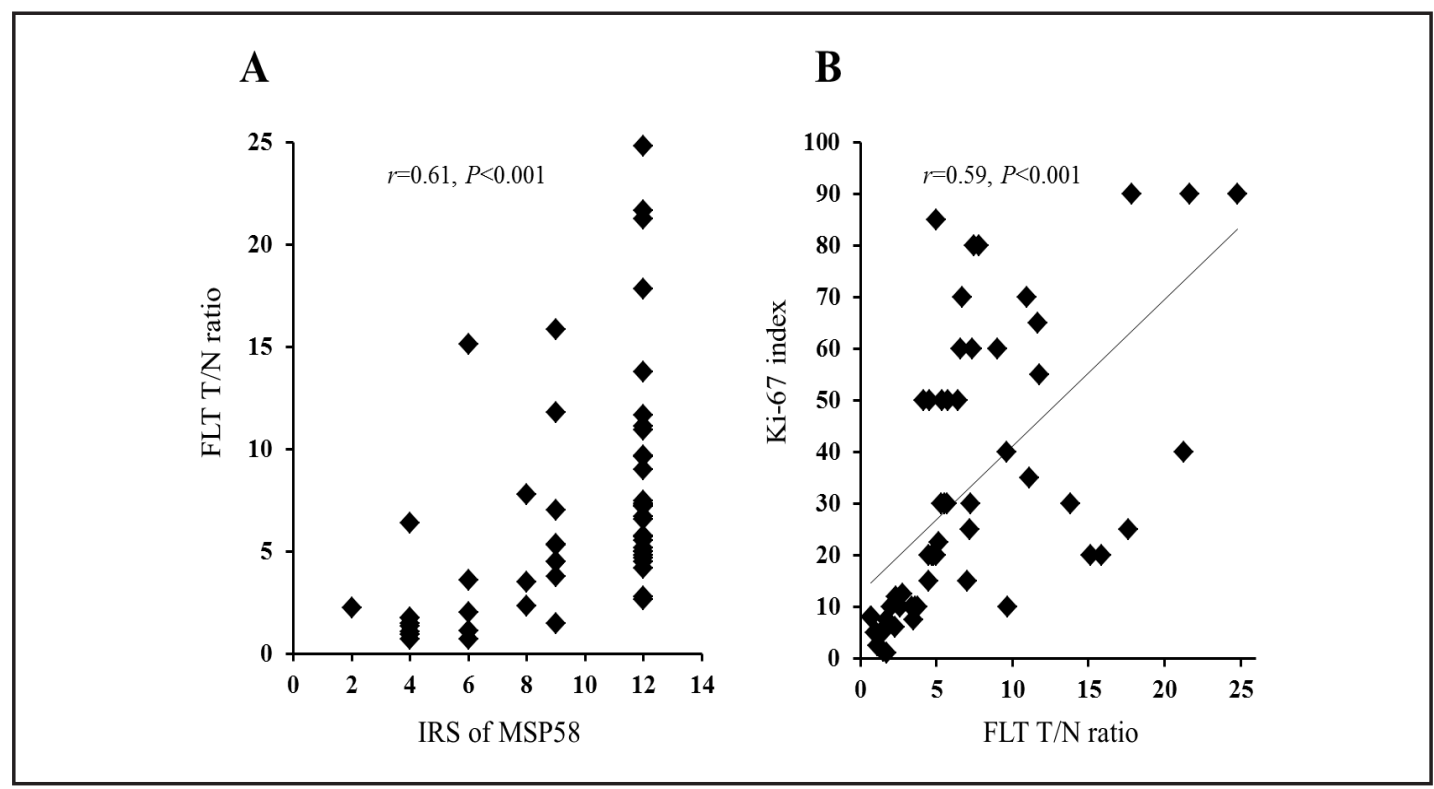

Fig. 5. (A) The IRS of MSP58 in the tumor shows a significant positive correlation with corresponding FLT $\mathrm{T} / \mathrm{N}$ ratio $(r=0.61, P<0.001)$. (B) The FLT T/N ratio of the tumor shows a significant positive correlation with the Ki-67 index $(r=0.59, P<0.001)$.

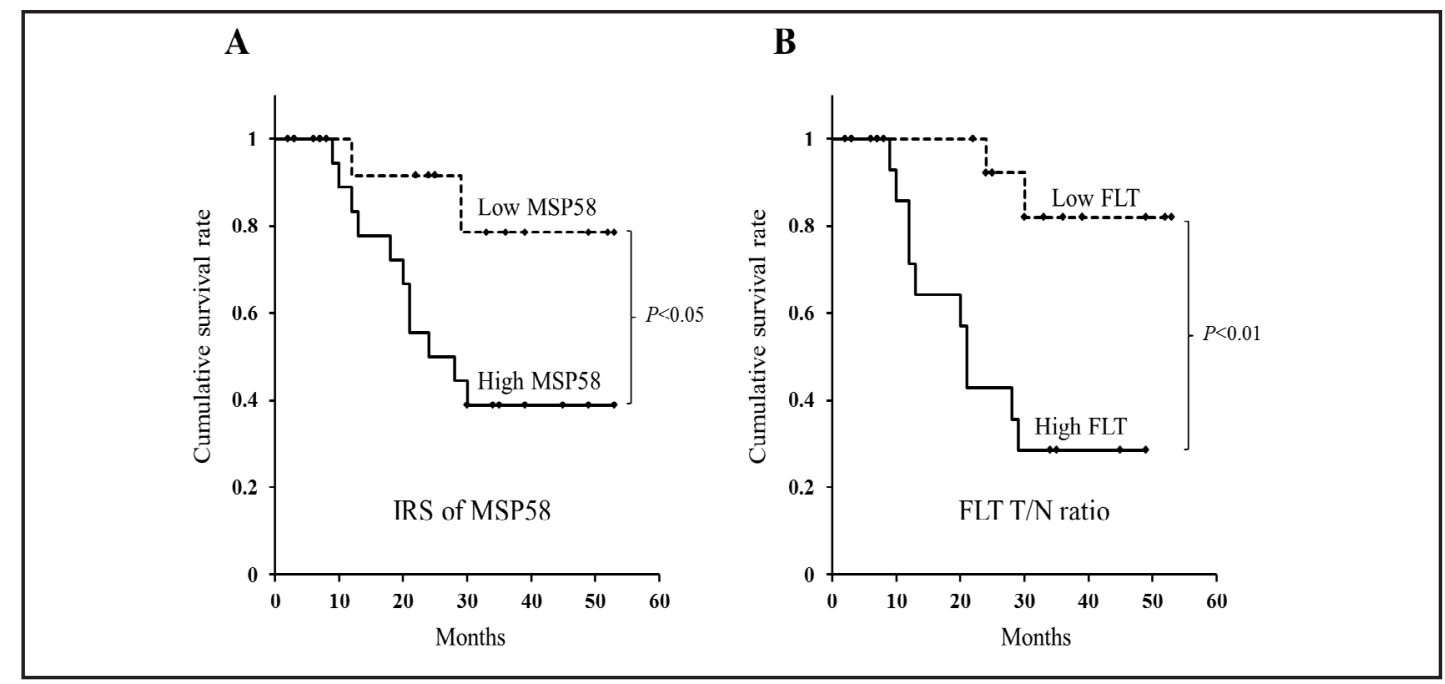

Fig. 6. Survival estimate was performed only in newly diagnosed tumors $(n=37)$ using Kaplan-Meier analysis. (A) The overall survival time in patients with low to moderate MSP58 expression (IRS of 2-8) was significantly longer than that with high MSP58 expression (IRS of 9-12) $(P<0.05)$. (B) The overall survival time in patients with low to moderate FLT T/N ratio $(\leq 5)$ was significantly longer than that with high $\mathrm{T} / \mathrm{N}$ ratio $(>5)(P<0.01)$.

different between grade III and grade IV gliomas $(\mathrm{P}<0.01)$, but grade II and grade III gliomas. The immunohistochemical expression of MSP58 in the tumor showed a highly significant positive correlation with corresponding FLT T/N ratio $(\mathrm{r}=0.61, \mathrm{P}<0.001$, non-parametric Spearman's rank test) (Fig. 5A). A linear regression analysis demonstrated a significant positive correlation between FLT T/N ratio and the Ki-67 index ( $r=0.59, \mathrm{P}<0.001)$ (Fig. 5B).

\section{MSP58 expression, PET uptake and survival}

Survival estimate was performed only in newly diagnosed tumors $(n=37)$. KaplanMeier analysis revealed a significant effect of MSP expression on the overall survival time $(P<0.05)$ (Fig. 6A). The median survival time was 24 months for patients with high MSP58 
expression (IRS of 9-12), whereas it was more than 53 months (undefined) for patients with low MSP58 expression (IRS of 2-8). Survival analysis showed a significant effect of FLT $\mathrm{T} / \mathrm{N}$ ratio on the overall survival time $(P<0.01)$ (Fig. 6B). The median survival time was 20 months for patients with high FLT T/N ratio (>5), whereas it was more than 53 months (undefined) for patients with low FLT T/N ratio $(\leq 5)$.

\section{Discussion}

This study demonstrates that MSP58 is overexpressed in almost all gliomas, especially in high grade gliomas. This result is in accordance with our previous study examined in 158 specimens of various grade of human gliomas [8]. The expression of MSP58 shows significant increase with tumor grade, with grade IV gliomas having the highest expression and is positively correlated with the Ki-67 index. The expression of MSP58 in the tumor also showed a highly significant positive correlation with corresponding FLT uptake value. This is the first study to demonstrate significant correlations among the expression of MSP58 and the Ki-67 index and PET molecular imaging for cell proliferation in human gliomas. Moreover, survival estimate reveals that the expression of MSP58 has a significant prognostic ability on the overall survival time similar to that provided by the uptake value of FLT-PET.

Gliomas are the mostcommon primary brain tumors arising in the central nervous system. Although various strategies have been proposed to improve the diagnostic and prognostic evaluation ability of the gliomas, a significant rate of misjudge still exists. Moreover, although the rapid development of multimodal therapeutic strategies over recent years, including surgical resection, local radiotherapy and systemic chemotherapy, the prognosis of gliomas, especially glioblastomas is still dismal. Therefore, the underlying mechanisms of the disease remain to be fully elucidated. Identification of some new key molecules and development of new methods that can help to diagnose glioma patients accurately have crucial significance for managing this terrible disease.

The nucleolar 58-kDa microspherule protein (MSP58) also referred to microspherule protein 1 (MCRS1), plays an important role in a variety of cellular processes including transcriptional regulation, cell proliferation and oncogenic transformation. It is a 462-amino acid protein that contains a nucleolar localization signal, a nuclear localization signal, a coiled-coil domain and a fork head-associated (FHA) domain that is also considered to be the phosphopeptide binding module $[1,2]$. The coiled-coil domain mediates the interactions with other proteins and confers mutual stabilization resistance to proteasomes-mediated degradation [18]. Proteins containing the FHA domain, such as Dun1, Rad53, Ki-67, NIPP1, Cds1 and Mek1, are all located in the nucleolus and are involved in multiple crucial cellular events, including the regulation of the cell cycle, DNA repair, transcription and mitosis [19, 20]. MSP58 also has been reported to interact with several proteins, such as nucleolar protein $\mathrm{p} 120$, Mi-2 $\beta$, transcription factors Daxx, DEC1, and also RNA-binding protein FMR [1, $2,21,22]$. These findings and structures suggest that MSP58 may have key transcriptional regulation functions in the nucleus and nucleolus.

Recently, MSP58 has also been reported to interact with telomerase transcriptional element- interacting factor (TEIF) and determine the effect of MSP58 on hTERT transcription. MSP58 was a negative regulator of hTERT expression and telomerase activity [23]. However, more and more accumulating evidence has identified the expression pattern of MSP58 is closely correlated with the tumor proliferation activity. It has been reported that downregulation of MSP58 inhibits the growth of human colorectal cancer cells via regulation of the cyclin D1-cyclin-dependent kinase 4-p21 pathway and has significant function in the carcinogenesis of colorectal carcinoma [4]. Their recent study showed that the expression of MSP58 in colorectal cancer specimens was closely correlated with poor overall survival time [24]. The immunohistochemistry results showed the expression level of MSP58 in hepatocellular carcinoma (HCC) was significantly higher than in adjacent normal tissues. MSP58si markedly reduced the proliferation and promoted the apoptosis of HepG2 and 
Huh7 cells and the Kaplan-Meier survival analysis in patients with HCC demonstrated that the survival time of the patients with low MSP58 expression was significantly longer than those with high expression during the 5-year follow-up period [6]. It was found that MSP58 was highly expressed in neuroblastoma tumor samples and cell lines and MSP58 targeted shRNA lentivirus attenuated neuroblastoma cell proliferation [25]. Our previous study also showed that MSP58 play an important role in glioma cell lines biological progression [7] and we further demonstrated that MSP58 expression correlates with the WHO grade and overall survival of patients with glioma [8]. These findings suggest that MSP58 has closely relationship with the malignancy and proliferation of glioma and might be a novel prognostic marker.

We are in an era of targeted therapy and molecular imaging. Several new tracers are being developed for use with PET to assess pathways that are altered in cancers, including energy use, cellular signaling, transport, and proliferation. Because increased proliferation is a hallmark of many cancers, several tracers have been examined to track the DNA synthesis pathway. Among them, 3'-deoxy-3'-[18F]fluorothymidine (FLT), a radio-labeled fluorinated thymidine analog, has emerged as a promising PET tracer for evaluating tumor biological activity in various tumors. FLT-PET has been shown to be useful for preoperative tumor grading in human gliomas [11-13]. Moreover, FLT is considered to be one of the most reliable biomarker of cell proliferation evaluated by the Ki-67 index [13]. FLT is phosphorylated by thymidine kinase-1 (TK-1), a principle enzyme in the salvage pathway of DNA synthesis and trapped inside the cells, although only trace amounts of FLT can be recovered from DNA extracts [26]. FLT is resistant to degradation and presumed not to cross back across the cell membrane, and is suitable for imaging with PET. The application of FLT phosphorylation as a biomarker of cell proliferation is based on the assumption that cellular FLT trapping after phosphorylation is a representation of thymidine incorporation into DNA [9]. TK-1 is expressed with the onset of the S-phase during DNA synthesis and decreased in non-dividing cells [27], and TK-1 activity is proportional to the rate of DNA synthesis and cell proliferation in tumors $[9,28]$. There are several reports attempting to relate FLT uptake to TK-1 expression in vitro and in animal models [29-31]. A recent study in human lung tumors demonstrated a significant correlation between FLT uptake and TK-1 immnunohistochemical expression [32]. However, it is noticeable that not all tumors with high proliferation have high uptake of FLT because multiple biological aspects including the blood-brain barrier disruption, nucleoside transport and metabolism are involved in FLT uptake in the tumor $[33,34]$.

\section{Conclusion}

The expression of MSP58 in the tumor showed a highly significant positive correlation with corresponding FLT uptake value. This is the first study to demonstrate significant correlations among the expression of MSP58 and the Ki-67 index and PET molecular imaging for cell proliferation in human gliomas. Moreover, survival estimate reveals that the expression of MSP58 has a significant prognostic ability on the overall survival time similar to that provided by the uptake value of FLT-PET. MSP58 will be one of the potential candidates targeting for glioma molecular therapy. FLT-PET might be used as an early measure of treatment response in proliferation-targeted therapy.

\section{Acknowledgements}

We gratefully acknowledge the excellent technical support of PET radiological technologist at our institution. This study was supported by a Grant-in-Aid for Scientific Research (B) (26293324) from the Ministry of Education, Science and Culture of Japan. Lin Wei is a research doctor from the Department of Neurosurgery, Xijing Hospital, Fourth Military Medical University, China. This study was also supported by a Grant of China (National Nature Science Foundation of China, No: 81001123 and No:81572469). 


\section{Cellular Physiology Cell Physiol Biochem 2016;38:635-645

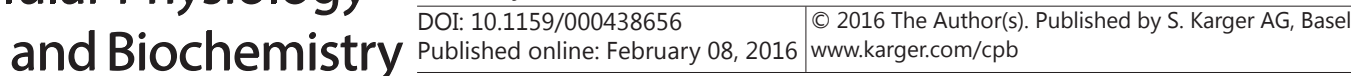 \\ Lin et al.: MSP58 and PET Imaging in Glioma Patients}

\section{Disclosure Statement}

The authors have no conflict of interest.

\section{References}

1 Ren Y, Busch RK, Perlaky L, Busch H: The 58-kDa microspherule protein (MSP58), a nucleolar protein, interacts with nucleolar protein p120. Eur J Biochem 1998;253:734-742.

2 Bader AG, Schneider ML, Bister K, Hartl M: TOJ3, a target of the v-Jun transcription factor, encodes a protein with transforming activity related to human microspherule protein 1 (MCRS1). Oncogene 2001;20:75247535.

3 Lin DY, Shih HM: Essential role of the 58-kDa microspherule protein in the modulation of Daxx-dependent transcriptional repression as revealed by nucleolar sequestration. J Biol Chem 2002;277:25446-25456.

4 Shi H, Chen S, Jin H, Xu C, Dong G, Zhao Q, Wang W, Zhang H, Lin W, Zhang J, Davidovic L, Yao L, Fan D: Downregulation of MSP58 inhibits growth of human colorectal cancer cells via regulation of the cyclin D1cyclin-dependent kinase 4-p21 pathway. Cancer Sci 2009;100:1585-1590.

5 Hsu CC, Lee YC, Yeh SH, Chen CH, Wu CC, Wang TY, Chen YN, Hung LY, Liu YW, Chen HK, Hsiao YT, Wang WS, Tsou JH, Tsou YH, Wu MH, Chang WC, Lin DY: 58-kDa microspherule protein (MSP58) is novel Brahmarelated gene 1 (BRG1)-associated protein that modulates p53/p21 senescence pathway. J Biol Chem 2012;287:22533-22548.

6 Zhong M, Zhang X, Li B, Chen CS, Ji GL, Li SX, Bi DQ, Zhao QC, Shi H: Expression of MSP58 in hepatocellular carcinoma. Med Oncol 2013;30:539.

7 Lin W, Zhang J, Liu X, Fei Z, Li X, Davidovic L, Tang Z, Shen L, Deng Y, Yang A, Han H, Zhang X, Yao L: RNAimediated inhibition of MSP58 decreases tumour growth, migration and invasion in a human glioma cell line. J Cell Mol Med 2009;13:4608-4622.

8 Lin W, Li XM, Zhang J, Huang Y, Wang J, Jiang XF, Fei Z: Increased expression of the 58-kD microspherule protein (MSP58) is correlated with poor prognosis in glioma patients. Med Oncol 2013;30:677.

9 Shields AF, Grierson JR, Dohmen BM, Machulla HJ, Stayanoff JC, Lawhorn-Crews JM, Obradovich JE, Muzik O, Mangner TJ: Imaging proliferation in vivo with [F-18]FLT and positron emission tomography. Nat Med 1998;4:1334-1336.

10 Toyohara J, Waki A, Takamatsu S, Yonekura Y, Magata Y, Fujibayashi Y: Basis of FLT as a cell proliferation marker: comparative uptake studies with [3H]thymidine and [3H]arabinothymidine, and cell-analysis in 22 asynchronously growing tumor cell lines. Nucl Med Biol 2002;29:281-287.

11 Shields AF: PET imaging with 18F-FLT and thymidine analogs: promise and pitfalls. J Nucl Med 2003;44:1432-1434.

12 Chen W, Cloughesy T, Kamdar N, Satyamurthy N, Bergsneider M, Liau L, Mischel P, Czernin J, Phelps ME, Silverman DH: Imaging proliferation in brain tumors with 18F-FLT PET: comparison with 18F-FDG. J Nucl Med 2005;46:945-952.

13 Choi SJ, Kim JS, Kim JH, Oh SJ, Lee JG, Kim CJ, Ra YS, Yeo JS, Ryu JS, Moon DH: [18F]3'-deoxy-3'fluorothymidine PET for the diagnosis and grading of brain tumors. Eur J Nucl Med Mol Imaging 2005;32:653-659.

14 Hatakeyama T, Kawai N, Nishiyama Y, Yamamoto Y, Sasakawa Y, Ichikawa T, Tamiya T: 11C-methionine (MET) and 18F-fluorothymidine (FLT) PET in patients with newly diagnosed glioma. Eur J Nucl Med Mol Imaging 2008;35:2009-2017.

15 Ullrich R, Backes H, Li H, Kracht L, Miletic H, Kesper K, Neumaier B, Heiss WD, Wienhard K, Jacobs AH: Glioma proliferation as assessed by 3'-fluoro-3'-deoxy-L-thymidine positron emission tomography in patients with newly diagnosed high-grade glioma. Clin Cancer Res 2008;14:2049-2055.

16 MacHulla HK, Schonermarck U, Schaaf A, Muller LP, Kloss C, Kruger J, Kunze G, Schonermarck G, Langner J: HLA-A, B, Cw and DRB1, DRB3/4/5, DQB1, DPB1 frequencies in German immunoglobulin A-deficient individuals. Scand J Immunol 2000;52:207-211.

17 Cao L, Cao W, Zhang W, Lin H, Yang X, Zhen H, Cheng J, Dong W, Huo J, Zhang X: Identification of 14-3-3 protein isoforms in human astrocytoma by immunohistochemistry. Neurosci Lett 2008;432:94-99. 


\section{Cellular Physiology Cell Physiol Biochem 2016;38:635-645

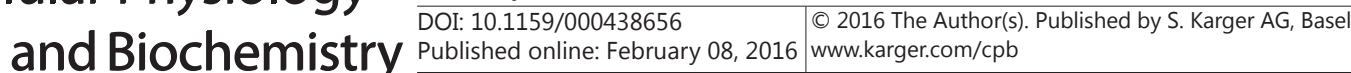 \\ Lin et al.: MSP58 and PET Imaging in Glioma Patients}

18 Maekawa T, Cheng W, Spiridon LN, Toller A, Lukasik E, Saijo Y, Liu P, Shen QH, Micluta MA, Somssich IE, Takken FL, Petrescu AJ, Chai J, Schulze-Lefert P: Coiled-coil domain-dependent homodimerization of intracellular barley immune receptors defines a minimal functional module for triggering cell death. Cell Host Microbe 2011;9:187-199.

19 Sun Z, Fay DS, Marini F, Foiani M, Stern DF: Spk1/Rad53 is regulated by Mec1-dependent protein phosphorylation in DNA replication and damage checkpoint pathways. Genes Dev 1996;10:395-406.

20 Bork P, Hofmann K, Bucher P, Neuwald AF, Altschul SF, Koonin EV: A superfamily of conserved domains in DNA damage-responsive cell cycle checkpoint proteins. FASEB J 1997;11:68-76.

21 Shimono K, Shimono Y, Shimokata K, Ishiguro N, Takahashi M: Microspherule protein 1, Mi-2beta, and RET finger protein associate in the nucleolus and up-regulate ribosomal gene transcription. J Biol Chem 2005;280:39436-39447.

22 Ivanova AV, Ivanov SV, Lerman ML: Association, mutual stabilization, and transcriptional activity of the STRA13 and MSP58 proteins. Cell Mol Life Sci 2005;62:471-484.

23 Hsu CC, Chen CH, Hsu TI, Hung JJ, Ko JL, Zhang B, Lee YC, Chen HK, Chang WC, Lin DY: The 58-kda microspherule protein (MSP58) represses human telomerase reverse transcriptase (hTERT) gene expression and cell proliferation by interacting with telomerase transcriptional element-interacting factor (TEIF). Biochim Biophys Acta 2014;1843:565-579.

24 Shi H, Li SJ, Zhang B, Liu HL, Chen CS: Expression of MSP58 in human colorectal cancer and its correlation with prognosis. Med Oncol 2012;29:3136-3142.

25 Wu L, Zhang ZG, Qin HZ, Zhang J, Gao GD, Lin W, Wang J: Downregulation of MSP58 suppresses cell proliferation in neuroblastoma cell lines. Neuroreport 2012;23:932-936.

26 Seitz U, Wagner M, Neumaier B, Wawra E, Glatting G, Leder G, Schmid RM, Reske SN: Evaluation of pyrimidine metabolising enzymes and in vitro uptake of 3'-[(18)F]fluoro-3'-deoxythymidine ([(18)F]FLT) in pancreatic cancer cell lines. Eur J Nucl Med Mol Imaging 2002;29:1174-1181.

27 Sherley JL, Kelly TJ: Regulation of human thymidine kinase during the cell cycle. J Biol Chem 1988;263:8350-8358.

28 Jacobs AH, Thomas A, Kracht LW, Li H, Dittmar C, Garlip G, Galldiks N, Klein JC, Sobesky J, Hilker R, Vollmar S, Herholz K, Wienhard K, Heiss WD: 18F-fluoro-L-thymidine and 11C-methylmethionine as markers of increased transport and proliferation in brain tumors. J Nucl Med 2005;46:1948-1958.

29 Rasey JS, Grierson JR, Wiens LW, Kolb PD, Schwartz JL: Validation of FLT uptake as a measure of thymidine kinase-1 activity in A549 carcinoma cells. J Nucl Med 2002;43:1210-1217.

30 Schwartz JL, Tamura Y, Jordan R, Grierson JR, Krohn KA: Monitoring tumor cell proliferation by targeting DNA synthetic processes with thymidine and thymidine analogs. J Nucl Med 2003;44:2027-2032.

31 Barthel H, Perumal M, Latigo J, He Q Brady F, Luthra SK, Price PM, Aboagye EO: The uptake of 3'-deoxy-3'[18F]fluorothymidine into L5178Y tumours in vivo is dependent on thymidine kinase 1 protein levels. Eur J Nucl Med Mol Imaging 2005;32:257-263.

32 Brockenbrough JS, Souquet T, Morihara JK, Stern JE, Hawes SE, Rasey JS, Leblond A, Wiens LW, Feng Q, Grierson J, Vesselle H: Tumor 3'-deoxy-3'-(18)F-fluorothymidine ((18)F-FLT) uptake by PET correlates with thymidine kinase 1 expression: static and kinetic analysis of (18)F-FLT PET studies in lung tumors. J Nucl Med 2011;52:1181-1188.

33 Shinomiya A, Kawai N, Okada M, Miyake K, Nakamura T, Kushida Y, Haba R, Kudomi N, Yamamoto Y, Tokuda M, Tamiya T: Evaluation of 3'-deoxy-3'-[18F]-fluorothymidine (18F-FLT) kinetics correlated with thymidine kinase-1 expression and cell proliferation in newly diagnosed gliomas. Eur J Nucl Med Mol Imaging 2013;40:175-185.

34 Shinomiya A, Miyake K, Okada M, Nakamura T, Kawai N, Kushida Y, Haba R, Kudomi N, Tokuda M, Tamiya T: 3'-Deoxy-3'-[(18)F]-fluorothymidine ([(18)F]-FLT) transport in newly diagnosed glioma: correlation with nucleoside transporter expression, vascularization, and blood-brain barrier permeability. Brain Tumor Pathol 2013;30:215-223. 